\section{La Révolution française}

Cahiers de l'Institut d'histoire de la Révolution française

22 | 2022

L'étranger en révolution(s)

\title{
Une Révolution pour tous? Comment des femmes anglaises ont vécu et écrit la Révolution française : le cas de Mary Wollstonecraft et Helen Maria Williams
}

Valentina Altopiedi

\section{OpenEdition}

\section{Journals}

Édition électronique

URL : https://journals.openedition.org//rf/6206

DOI : $10.4000 /$ Irf.6206

ISSN : 2105-2557

Éditeur

IHMC - Institut d'histoire moderne et contemporaine (UMR 8066)

Référence électronique

Valentina Altopiedi, « Une Révolution pour tous ? Comment des femmes anglaises ont vécu et écrit la Révolution française : le cas de Mary Wollstonecraft et Helen Maria Williams », La Révolution française [En ligne], 22 | 2022, mis en ligne le 20 janvier 2022, consulté le 23 janvier 2022. URL : http:// journals.openedition.org/lrf/6206 ; DOI : https://doi.org/10.4000/lrf.6206

Ce document a été généré automatiquement le 23 janvier 2022.

(c) La Révolution française 


\title{
Une Révolution pour tous?
}

Comment des femmes anglaises ont vécu et écrit la Révolution française : le cas de Mary Wollstonecraft et Helen Maria

\section{Williams}

\author{
Valentina Altopiedi
}

1 Le bouleversement de la Révolution française a secoué l'Europe entière. La société britannique est sans doute celle qui a le plus ressenti les conséquences de ces événements : d'une part la longue rivalité politique et militaire de la « Seconde guerre de Cent Ans" incitait à regarder avec intérêt les problèmes et l'humiliation des Bourbons, d'autre part le danger d'une contamination révolutionnaire animait le débat politique anglais et conduisait la majorité de la population ainsi que de l'élite politique à désapprouver les événements d'outre-Manche ${ }^{1}$. Parmi les Honest Whigs et Dissenters qui avaient embrassé avec enthousiasme le déclenchement révolutionnaire, Richard Price, dont le Discourse on the Love of Our Country ${ }^{2}$ avait été durement critiqué par Edmund Burke dans Reflections on the Revolution in France ${ }^{3}$, déclencha une des plus féroces controverses politiques anglaises depuis la Glorieuse Révolution. Le trinôme Price, Burke, Paine ne doit cependant pas dissimuler la richesse des contributions et interventions des hommes et des femmes anglaises qui attaquèrent ou défendirent la Révolution française. Mary Wollstonecraft et Helen Maria Williams n'ont pas seulement joué un rôle important dans l'interprétation de la Révolution, mais aussi sur le front de la participation en décidant de s'installer à Paris pour voir de leurs propres yeux les événements de France. Étudier comment Mary Wollstonecraft et Helen Williams ont décrit et vécu la Révolution française permet de réfléchir à la construction du récit de la Révolution au prisme du regard de femme et d'étrangère et de revendiquer 
l'agentivité de celles qui, comme Wollstonecraft et Williams, ont voyagé, écrit et pris la parole pour défendre leurs droits.

\section{Écrire pour changer la société : Mary Wollstonecraft, Helen Maria Williams et la littérature éducative à la veille de la Révolution française}

2 Parmi les contes que l'institutrice Madame Mason, alter ego de Mary Wollstonecraft et protagoniste de ses Original Stories, raconte à ses élèves pour leur apprendre à bien traiter les animaux et à se méfier de la cruauté des hommes, on trouve l'histoire de Paul Pellisson qui, emprisonné à la Bastille, avait trouvé du réconfort en compagnie d'une araignée :

Je vous l'ai dit, Robin était enfermé dans une prison. En France, ils en ont une terrible. Les pauvres misérables qui y sont enfermés vivent entièrement seuls; ils n'ont pas le plaisir de voir des hommes ou des animaux; ils n'ont pas non plus le droit d'avoir des livres. Ils vivent dans une solitude sans confort [...] Un captif misérable a trouvé une araignée, il l'a nourrie pendant deux ou trois ans; elle s'est laissée apprivoiser et a pris part à son repas solitaire. Son gardien l'a observé, et a mentionné ce fait à un supérieur, qui lui a ordonné de l'écraser. En vain l'homme a supplié que son araignée soit épargnée $e^{4}$.

Cette petite anecdote publiée en 1788 permet de bien introduire la pensée de la philosophe anglaise à la veille de la Révolution : le thème de l'éducation, qui, dans cette anecdote, s'exprime par le respect des animaux, et, en particulier, la nécessité d'améliorer l'éducation des filles et des femmes pour réformer la société marque presque toute la production littéraire de Mary Wollstonecraft ${ }^{5}$.

Original Stories est un ouvrage de littérature enfantine publié un an après le manuel de conduite pour filles intitulé Thoughts on the Education of Daughters ${ }^{6}$. Sur le modèle d'Adèle et Théodore $e^{7}$ par madame de Genlis, dont Wollstonecraft appréciait la posture intellectuelle, mais à qui elle reprochait ses préjugés nobiliaires, Original Stories décrit la rééducation de deux filles de douze et quatorze ans chez madame Mason après la mort de leur mère. À travers les contes moraux et les excursions pédagogiques organisés par leur institutrice, Marie et Caroline perdent les défauts qu'elles avaient développés dans leur enfance et prennent le chemin de la transformation en femmes vertueuses. Alors que le premier effort littéraire de Wollstonecraft, Thoughts on the Education of Daughters, dans lequel elle reprenait le style et la rhétorique des manuels de conduite, avait connu un très modeste succès, Original Stories a été très bien accueilli par le public anglais du dix-huitième siècle au point que la première édition en 1788 a été suivie par une deuxième en 1792, illustrée par William Blake, et a continué d'être imprimée jusque dans les années 1920 , traduite en allemand en $1795^{8}$ et en français en $1799^{9}$.

Dans ces deux premiers ouvrages de Mary Wollstonecraft, on peut aisément reconnaître l'influence de Locke $^{10}$ dans le rôle des sens dans le processus d'apprentissage et celle de Rousseau (bien que l'auteure blâmait le philosophe genevois pour ses préjugés exposés dans l'Émile ou De l'éducation à propos de la prétendue faiblesse des femmes ${ }^{11}$ ) dans la nécessité d'enseigner par l'exemple et l'expérience plutôt que par les préceptes, mais on y lit surtout l'écho de l'expérience de Mary Wollstonecraft en tant qu'éducatrice et institutrice ${ }^{12}$. En effet, au début des années 1790, Mary Wollstonecraft avait fondé avec ses sœurs, Eliza et Everina, ainsi qu'avec 
son amie Fanny Blood une école à Newington Green, district des environs de Londres et siège d'une communauté dissenter bien établie. Bien que l'expérience de l'école de Newington Green puisse être considérée éphémère du fait de son échec survenu deux ans et demi après son ouverture, ce parcours a cependant, comme le souligne Janet Todd $^{13}$, consacré Wollstonecraft en tant que pédagogue, puisqu'elle a formulé sa propre théorie de l'éducation. Par ailleurs, dans Original Stories, on trouve des références claires à la brève carrière d'institutrice privée que Wollstonecraft avait eue en Irlande auprès de deux filles d'une famille anglo-irlandaise après la fermeture de son école (les personnages principaux ont, en fait, les mêmes prénoms que les filles de la famille Kingsborough).

6 L'échec de l'école de Newington Green et sa brève expérience d'institutrice ont très probablement persuadé Mary Wollstonecraft de laisser de côté la carrière d'éducatrice et de prendre la plume pour écrire sur le thème de l'éducation. Déçue par les maigres possibilités de carrière réservées aux femmes, Wollstonecraft entrevoit dans l'écriture tout d'abord une voie pour s'émanciper de façon économique, puis un moyen de promouvoir un nouveau modèle de société dans laquelle la femme puisse jouer un rôle non subordonné à l'homme. En novembre 1787, inquiète de sa situation financière ainsi que de celle de ses sœurs après la fermeture de l'école, Wollstonecraft avoue à Everina son espoir et sa détermination de faire de l'écriture une véritable profession, grâce au support de son éditeur, Joseph Johnson : «Mr Johnson dont la gentillesse peu commune m'a sauvée, je crois, du désespoir [...], m'assure que, si j'exerce mes talents pour l'écriture, je pourrais vivre de manière confortable. Je vais donc être la première d'un nouveau genre ${ }^{14} »$.

7 Wollstonecraft se propose donc d'ouvrir la carrière des lettres, en tant que profession, aux femmes, mais, en même temps, elle se positionne dans le sillage de la littérature pour enfants qui, comme a suggéré Mitzi Myers ${ }^{15}$, était, entre le dix-huitième et dixneuvième siècle, le genre littéraire qui permettait aux femmes de promouvoir une vision différente de la société. D’ailleurs, il faut rappeler ici que le début de la carrière littéraire de Wollstonecraft, soutenue par l'éditeur libéral Joseph Johnson, coïncide avec la fréquentation des intellectuels dissenters de Newington Green, qui en refusant de se conformer à l'église anglicane (d'où l'appellation de dissenter) voyaient justement dans l'éducation la pierre angulaire de tout mouvement de réforme sociale.

8 À la veille de la Révolution française, cette volonté de faire évoluer l'éducation pour reformer la société est très présente dans la production littéraire de Mary Wollstonecraft: elle est très clairement expliquée, au-delà des textes éducatifs proprement dit comme Thoughts on the Education of Daughters et Original Stories, dans le roman Mary: A Fiction ${ }^{16}$ et dans les comptes-rendus que Wollstonecraft écrit à partir de 1788 pour l'Analytical Review, dans lesquels elle exprime également un intérêt croissant pour les événements qui se déroulent en France. Dans les comptes-rendus publiés dans la revue à laquelle Wollstonecraft collabore suite à l'invitation de son ami et éditeur Johnson, on trouve, outre l'opposition évidente aux romans sentimentaux de femmes que Wollstonecraft attaque avec un esprit mordant ${ }^{17}$, les traces de sa participation au milieu dissenter anglais. C'est dans l'Analytical Review que Wollstonecraft recense en janvier 1789 sa propre traduction anglaise de De l'importance des opinions religieuses ${ }^{18}$ par Jacques Necker et, en décembre 1789, A Discours on the Love of Our Country ${ }^{19}$ par Richard Price, avant la publication de la réplique par Edmund Burke. Wollstonecraft loue le style et le langage simple, clair et éloquent de Price et rend hommage à la définition 
d'amour pour son propre pays élaborée par le philosophe anglais : «l'amour, le fruit de la raison, et non les pulsions non-maîtrisées de la nature, qui tendent toujours à des extrêmes égoïstes ${ }^{20}$. Dans une longue citation de Price, la philosophe salue l'époque dans laquelle « la lumière [que tous les amis de la liberté] ont envoyé, après avoir rendu l'AMÉRIQUE libre, s'est reflétée en FRANCE, et là s'est transformée en une flamme qui a réduit le despotisme en cendres, et qui réchauffe et éclaire l'EUROPE $»^{21}$.

De même que la Révolution fait irruption dans la production littéraire et dans la vie de Mary Wollstonecraft, elle survient de façon analogue dans celle de Helen Maria Williams. C'est d'ailleurs la prophétie de la destruction de la Bastille, contenue dans le roman sentimental $J_{u l i i^{22}}$, qui relie de façon symbolique la production sentimentale de l'auteure avec la littérature engagée. Née à Londres en 1761 dans une famille d'origine écossaise, Helen Maria Williams établit rapidement sa réputation littéraire grâce à un recueil de poèmes publié en 1786 . Poétesse bien connue de l'age of sensibility, Williams entre dans le monde littéraire de Londres en fréquentant, entre autres, Elizabeth Montagu, fondatrice de la Blue Stockings Society, et Anna Seward, la célèbre poétesse connue sous le surnom de "cygne de Lichfield». Si les biographes de Williams ${ }^{23}$ distinguent habituellement, dans l'itinéraire biographique et littéraire de l'auteure, la phase de la poétique de la sensibilité de celle de la littérature engagée stimulée par la Révolution française, il faut noter que les poèmes de Williams sont déjà emprunts d'un contenu politique. Parmi les thèmes récurrents, on trouve, par exemple, celui des effets dévastateurs des guerres (Edwind et Eltruda se déroule pendant la guerre des Deux Roses, An American Tale pendant la guerre d'Indépendance américaine, tandis que Peru traite du massacre espagnol des Incas). De plus, à partir de 1788 avec A Poem on the Bill Lately Passed for Regulating the Slave Trade ${ }^{24}$, Williams collabore directement à la campagne abolitionniste anglaise: encouragée par Andrew Kippis, élu au London Committee for Abolition, Williams compose un sonnet en relation avec le Slave Trade Regulation Act voté pendant l'été 1788 pour limiter la taille des navires négriers. D'ailleurs, Williams elle-même a raconté dans ses Souvenirs qu'elle avait amené en France plusieurs exemplaires d'un texte programmatique du comité de Londres, Description of a Slave Ship, et de nombreux camées de Wedgwood, représentant l'esclave noir enchaîné, symbole international de la lutte contre la traite des esclaves : «J'ai distribué, lors de mon premier séjour à Paris, plusieurs gravures d'un plan des bateaux négriers, et plusieurs camées de Wedgwood, représentant l'esclave noir à genoux, plaidant sa cause et montrant ses chaînes ${ }^{25}$.

10 À partir de 1790, Williams, comme Mary Wollstonecraft, salue avec enthousiasme le déclenchement de la Révolution française : dans le roman $J u l i a^{26}$, elle loue la prise de la Bastille comme le jour glorieux qui donne naissance à une époque nouvelle. Julia, inspiré entre autres par Julie, ou la Nouvelle Héloïse ${ }^{27}$ de Rousseau, peut d'une certaine façon représenter le prototype du livre de conduite pour filles (les lectrices sont, en effet, appelées à suivre l'engagement de la protagoniste dans la lecture, dans l'étude de la musique et dans la générosité envers autrui). C'est en réalité un roman qui quitte la sphère domestique pour embrasser l'actualité politique et réfléchir sur la société contemporaine. Le roman se déroule en Angleterre au moment de la guerre d'Indépendance américaine et contient un poème sur la Bastille et la prophétie de sa destruction: Williams peint un prisonnier de la Bastille qui se demande si la Terre pourra voir un jour plus glorieux que celui où «des millions qui, d'une seule voix, 
réclamaient les droits de l'humanité $»^{28}$ [millions with according mind,/Who claim the rights of human kind], après avoir pris d'assaut la prison détestée.

\section{La Révolution, aube d'une nouvelle ère?}

11 Helen Maria Williams, comme Mary Wollstonecraft, était une auteure bien établie et intégrée dans les cercles dissenters anglais lorsque la nouvelle de la prise de la Bastille atteignit l'Angleterre ${ }^{29}$ : Deborah Kennedy ${ }^{30}$ estime que le Discourse on the Love of Our Country $^{31}$ par Richard Price a été débattu précisément chez elle. En juin 1790, justement en raison de son intérêt pour les événements qui avaient lieu en France, Williams commença son séjour à Paris en compagnie de sa mère et de sa sœur. Les célébrations à l'occasion de la Fête de la Fédération ont été le premier événement solennel auquel Williams a assisté et qu'elle a décrit de façon enthousiaste dans ses Lettres de France, dont l'histoire de la publication est particulièrement intéressante pour commencer à enquêter sur le récit de la Révolution, mais aussi pour son rôle dans la France thermidorienne.

Entre 1790 et 1796, Helen Maria Williams écrit et publie en anglais entre Londres et Dublin huit volumes de Letters from France ${ }^{32}$. Les huit volumes, mêlant l'écriture de voyage et la forme épistolaire, décrivent les événements dont Williams elle-même avait été témoin entre juillet 1790 et août 1795 ; ils ont connu un succès remarquable sur le marché éditorial de l'Angleterre de la fin du siècle. Cependant, seuls deux volumes ont été traduits en français: le premier, publié peu après les événements qu'il décrivait, dans le but de convaincre le public anglais de soutenir la cause de la Révolution, et le cinquième, publié en 1795 pour décrire la «tyrannie de Robespierre». Le premier volume racontait ce qui s'était passé en France entre juillet et septembre 1790 et incluait l'histoire paradigmatique de monsieur du Fossé, dont l'histoire d'amour, empêchée sous l'Ancien Régime, était justement rendue possible par le déclenchement de la Révolution. Traduit en français en 1791, Les lettres écrites en France à une amie en Angleterre pendant l'année $1790^{33}$ sont un exemple limpide d'une écriture qui, mêlant le style épistolaire et le langage sentimental, s'inscrit cependant entièrement dans le débat politique de l'époque, en particulier dans les controverses anglaises sur la Révolution française. L'objectif de Williams était, en fait, celui de défendre la cause de la Révolution aux yeux effrayés du public d'outre-Manche : c'est le même traducteur, Pierre de La Montagne, qui définit, dans une de ses interventions au cours de la traduction française, l'œuvre de Williams comme « une réponse complète à tous les sophismes de M. Burke $»^{34}$.

13 L'ouvrage s'ouvre avec la description de la Fête de la Fédération comme "le plus beau spectacle qui peut-être ait jamais été représenté sur le vaste théâtre du monde ${ }^{35}$. Williams peint la magnificence et la sublimité de l'événement, mais insiste surtout sur le rôle de la nation, qui constitue à son avis le vrai spectacle :

Comment rendre les sensations impétueuses, l'ivresse et la joie de cette immense multitude, un demi-million d'hommes contemplant un spectacle qui offroit à leurs regards tout ce qui peut élever et flatter l'esprit humain ; qui réunissoit aux idées morales les plus propres à exciter l'enthousiasme, la pompe et l'éclat d'une cérémonie religieuse qui parloit à l'imagination, au cœur et à la raison ? ${ }^{36}$

Williams décrit tout d'abord sa participation aux événements en tant que femme étrangère sur cette «terre de bonheur »: «je me réjouis de la joie de ses habitans; je 
joignis ma voix à ce concert universel, et de tout mon cœur, de toute mon âme, je crierai à l'unisson : vive, vive la nation ${ }^{37}$ !». Elle veut visiter la Bastille, symbole d'un gouvernement tyrannique qui pendant trop longtemps avait tenu le peuple français sous un joug insupportable, et déclare qu'elle ne pardonnera jamais à ceux qui, ayant vu les cellules de la prison, n'ont pas béni sa destruction.

15 En décrivant une séance de l'Assemblée nationale, à laquelle elle et sa sœur ont eu la chance d'être admises en tant que femmes et étrangères, Williams souligne la particularité de son point de vue et l'accueil favorable dont elle avait bénéficié : « nous n'avions aucune liaison avec cet officier, aucun droit à sa complaisance, que celui d'être femmes et étrangères. Mais ce sont là de tous les titres les plus puissans auprès de la politesse française $»^{38}$. D'ailleurs, elle applaudit au projet d'abolition de la traite et rappelle au public anglais l'opiniâtreté de la chambre des Communes qui s'obstine à conclure que ce qui est évidemment injuste en morale puisse être juste en politique, mais en même temps elle avertit que « les Africains n'ont pas longtemps à souffrir, le triomphe de leurs oppresseurs touche à son terme, l'Europe avance à pas rapides vers une époque trop éclairée pour que d'aussi monstrueux abus puissent subsister ${ }^{39}$. Williams demande aux Anglais de regarder sans préjugé les dispositions de l'Assemblée nationale française et accuse ses compatriotes de vouloir garder le monopole de la liberté. Elle se confronte aussi au thème de la violence révolutionnaire après avoir vu la lanterne à laquelle avaient été immolées les premières victimes de la Révolution, mais avoue que "malgré quelques exemples affreux de vengeance publique, la liberté de vingt-quatre millions d'hommes aura été achetée à un prix infiniment inférieur à ce que l'expérience et l'histoire nous donnoient le droit de l'attendre $»^{40}$.

16 Le premier volume de Letters from France se conclut avec la description de l'histoire des malheurs de monsieur du Fossé. Il s'agit, selon une logique qui devient très commune dans la littérature romanesque française après 1789 , de la célébration de la Révolution à travers le mariage entre le fils d'un noble et une fille roturière. Après avoir décrit les tortures, les pièges et les lettres de cachet que le baron utilise pour emprisonner son fils, Williams célèbre la Révolution qui permet enfin à monsieur du Fossé d'être «en sûreté dans son pays natal $\aleph^{41}$. Il n'est pas anodin que Williams se réjouit que son interlocutrice dans la fiction du roman épistolaire soit d'accord avec elle en estimant que l'histoire de monsieur du Fossé est une bonne raison pour aimer la Révolution: Williams elle-même admet que c'est le sentiment de l'amitié qui a fait passer son attention " des annales de l'imagination, à l'histoire de la politique ${ }^{42}$ et, en répondant à ceux qui auraient pu critiquer l'excessif enthousiasme de sa description de la Fête de la Fédération, elle rappelle qu'«il est très difficile de ne pas partager la félicité générale. C'est de cette sympathie que vient mon amour de la révolution française. Ma profession de foi politique est une affaire purement de cœur, il auroit été trop absurde de consulter ma tête sur des objets dont elle est aussi peu capable de juger sainement $\aleph^{43}$. Derrière cette profession de foi romantique, on ne doit pas voir l'exaltation des sentiments sur la raison, mais plutôt une référence à la morale des Lumières que Williams embrasse pour s'opposer à la traite des esclaves et pour défendre la liberté des femmes et des hommes français.

17 Dans le premier volume de ses Letters from France Williams défend la Révolution française et conclut que «c'est aux politiques à décider si les matériaux, dont la nouvelle constitution est composée, sont durables ou non; mais sans être doué d'une sagacité extraordinaire, on peut prédire que désormais les Français seront libres ${ }^{44}$. 
Cet éloge de la France engagée sur le chemin de la liberté se retrouve aussi dans le deuxième volume, publié à Londres en 1792 et jamais traduit en français, dans lequel Williams espère que «les authentiques principes de la liberté éclairée seront bientôt mieux compris, et pourraient peut-être être adoptés dans un avenir pas si lointain par toutes les nations de l'Europe. La Liberté pourrait ramener 'ses fils des pays lointains et ses filles des extrémités de la Terre' $»^{45}$. En citant le verset biblique d'Isaïe $(43: 6)$ qui a pour signification l'unification de tous les peuples de la Terre sous un seul Dieu, la dissenter Williams célébrait la liberté qui aurait unifié tous les peuples dans un rêve quasiment millénariste d'une république universelle.

Ce n'est certainement pas un hasard si le seul autre volume de Letters from France traduit en français en 1795 a été celui consacré à la période comprise entre le 31 mai 1793 et le 10 thermidor an II (28 juillet 1794). Le volume, tel qu'il avait été conçu par l'auteure, s'inscrivait parfaitement dans la logique de la réaction thermidorienne, mais, en même temps, il s'alignait sur l'esprit dominant en Angleterre en justifiant le soutien de l'auteure à la bonne Révolution de 1789 distinguée de celle épouvantable de l'an II. Cette fois encore, Williams adoptait la fiction littéraire de lettres envoyées à une amie en Angleterre et l'expérience autobiographique se mêlait au récit historique et politique. En fait, il ne faut pas oublier que Williams, en tant que sujet du roi George III, avait été emprisonnée entre le 9 octobre et le 4 décembre 1793, d'abord à la prison du Luxembourg, puis au couvent des Anglaises. Le volume, traduit peu après sa publication en anglais ${ }^{46}$, était construit autour de deux pôles opposés : le martyre de la Gironde et la construction de la figure de Robespierre comme tyran.

19 L'histoire s'ouvre sur la description de l'arrestation de la protagoniste et de sa sœur, mais, dès les premières lignes, il est clair qu'aux yeux de Williams le véritable traumatisme est celui de la journée du 2 juin 1793 marquée par l'arrestation des vingtneuf députés de la Convention ${ }^{47}$ avec lesquels l'auteure se sent étroitement liée: Williams souligne qu'elle avait été enfermée par le comité révolutionnaire dans la même pièce «que le représentant du peuple Valazé avoit occupé, et d'où il avoit été transféré depuis quelques semaines, dans la prison de la Conciergerie ${ }^{48}$. Le récit de l'emprisonnement au Luxembourg et la description des cellules dans lesquelles sont inscrits les noms de Cincinnatus, Brutus, Socrate servent non seulement à établir un lien entre l'auteure et les députés, mais surtout à exalter le républicanisme des députés qui, par « un respect porté à l'excès pour les droits des individus $»^{49}$ n'avaient pas pu arrêter la faction liberticide dirigée par Robespierre. Williams décrivait la sérénité avec laquelle Marc David, Alba Lasource et Charles Alexis Brûlart de Genlis (époux de madame de Genlis) avaient fait face à l'emprisonnement et au procès au «tribunal sanguinaire $\aleph^{50}$. Mais c'est au moment de l'exécution de la sentence de mort que les députés de la Gironde atteignent, selon la description de Williams, le statut mythique et héroïque des martyrs républicains : l'auteure, en reprenant l'autoreprésentation déjà adoptée par les mêmes députés (et bien analysée par Michel Biard, Harold T. Parker et Dorinda Outram ${ }^{51}$ ), décrit "le calme de l'innocence $»^{52}$ avec lequel «les fondateurs et les plus zélés défenseurs de la République ne tardèrent pas à terminer leur vie sur l'échafaud " ${ }^{53}$. Les condamnés affrontent la mort avec le courage de l'innocence : « nous marchions à grands pas, l'âme triomphante de voir qu'une belle mort ne manquoit pas à de si belles vies, et qu'ils remplissoient d'une manière digne d'eux la seule tâche qu'il leur restât à remplir, celle de bien mourir $\aleph^{54}$. 
20 En décrivant les actions du tribunal révolutionnaire et les condamnations du « règne de la Terreur ", Williams adopte une rhétorique, typique du moment post-thermidorien, qui isole dans la figure de Robespierre l'éminent responsable du chaos qui a bouleversé la France. Il est significatif de noter qu'après une brève description de l'arrivée au pouvoir de l'avocat artésien, Williams insiste surtout sur des accusations personnelles contre le "tyran $»^{55}$. En accord avec d'autres analyses comme La vie et les crimes de Robespierre ${ }^{56}$ de l'abbé Proyart, Williams évoque la jeunesse du député pour montrer que ses premiers crimes découlaient de son envie et son ambition personnelle. Selon l'auteure, la classe la plus poursuivie par le tyran était, en fait, celle des hommes de lettres, « contre lesquels son âme atroce réunissoit la jalousie de la rivalité aux fureurs de la tyrannie $»^{57}$. Il est évident que Williams elle-même se sentait incluse dans la liste des victimes de Robespierre précisément en raison de son talent et de son activité d'écrivaine : elle faisait référence aux deux volumes publiés anonymement en anglais (mais qui lui furent bientôt attribués), dans lesquels il était «tracé le portrait du tyran avec les traits et le sombre coloris qui convenoient à son hideux caractère ${ }^{58}$. Dans l'été 1794, avant le 9 thermidor (27 juillet), Williams quitta la France et s'installa en Suisse, en compagnie de son éditeur et amant John Hurford Stone (président de la Société des Amis des Droits de l'Homme), où elle resta six mois, recueillant ce qui fera la matière de son livre A Tour in Switzerland; Or, a View of the Present State of the Government and Manners of those Cantons: with Comparative Sketches of the Present State of Paris ${ }^{59}$, publié à Londres en 1798 et traduit en français la même année ${ }^{60}$. D'ailleurs la liaison professionnelle et personnelle de Williams avec le fondateur et directeur de l'imprimerie anglaise de Paris questionne la collaboration de l'écrivaine anglaise avec le gouvernement révolutionnaire de l'an II ; en effet, dans une lettre envoyée en février 1794, Stone annonçait fièrement à son frère la publication d'une revue nationale dont Williams aurait dû être la rédactrice en chef ${ }^{61}$ et une lettre de Nicholas Madgett, chef du bureau des traductions rattaché au Comité de salut public, signale qu'elle avait été engagée par Robespierre lui-même pour traduire la version séminale de son discours Sur les principes de la morale politique qui doivent guider la Convention nationale dans l'administration intérieure de la République ${ }^{62}$.

21 L'itinéraire de Mary Wollstonecraft dans la France de la Révolution, s'il suit une chronologie légèrement différente de celui d'Helen Maria Williams, retrace de façon générale celui de la romancière dissenter, surtout à l'égard de la communauté d'expatriés anglophones présents à Paris. Bien qu'intéressée par les événements de France de l'été 1789, comme le montre le compte-rendu du discours de Price publié dans l'Analytical Review, Wollstonecraft ne s'installe à Paris qu'à partir de décembre 1792 et reste en France jusqu'en avril 1795. Certes, par rapport à Williams, Wollstonecraft ne subit pas le décret d'arrestation contre les sujets du roi George III, parce que son amant, Gilbert Imlay, l'avait déclarée à l'ambassade américaine comme sa femme en août 1793. Wollstonecraft s'inscrit dans une communauté bien établie d'Anglais à Paris et participe aux réunions à l'hôtel White ${ }^{63}$. Helen Maria Williams, qui se trouvait dans la capitale depuis l'été 1790 et qui était reconnue comme une écrivaine dévouée à la cause de la Révolution, en faisait également partie: elle était même présente, avec John Hurford Stone, lors du « festin patriotique » fondateur de la Société des Amis des Droits de l'Homme le 18 novembre 1792 à l'hôtel White ${ }^{64}$. Le 21 novembre 1792, Le Patriote français de Brissot rapporte les «toasts portés dans une société d'Anglais, réunis chez le restaurateur White pour célébrer la liberté française et le succès de ses armes ${ }^{65}$. Le onzième toast est porté " aux dames de la Grande-Bretagne, 
et particulièrement à celles qui se sont distinguées par leurs écrits pour la révolution française, Mme Smith, miss H. M. Williams " ${ }^{66}$. Si l'hommage à Williams souligne le rôle joué par sa présence à Paris pendant la Révolution, Charlotte Turner Smith avait écrit et publié ses ouvrages en faveur de la Révolution, comme Mary Wollstonecraft, sans se déplacer en France, mais, à la différence de cette dernière, elle avait utilisé le genre littéraire du roman (considéré le plus propre à l'écriture des femmes), alors que Wollstonecraft avait embrassé une écriture délibérément neutre. Alors que Smith était célébrée pour son roman Desmond ${ }^{67}$, publié en juin 1792 et traduit en français ${ }^{68}$ en 1793, dans lequel, par le biais de son jeune protagoniste qui visitait la France entre juin 1790 et février 1792, elle détruisait la représentation féroce de la Révolution et des révolutionnaires, $A$ Vindication of the Rights of $\mathrm{Men}^{69}$, qui était la première ${ }^{70}$ de quarantecinq réponses aux accusations que Burke avait porté contre la Révolution française, n'était pas mentionné dans les toasts. À ce propos, il faut préciser que le nom de Wollstonecraft n'est même pas cité dans le compte-rendu bien plus approfondi du dîner publié dans le Morning Chronicle ${ }^{71}$ et écrit presque certainement par Stone lui-même ; cette absence pourrait être due au mépris de ce dernier pour Wollstonecraft qui, très significativement, était surnommée par le couple Stone-Williams « rights of women ${ }^{72}$.

Probablement initié comme un long compte-rendu de Reflections on the Revolution in France de Burke, A Vindication of the Rights of Men est un texte dans lequel la réflexion politique se mélange avec le langage mordant, que Wollstonecraft avait déjà utilisé dans ses comptes-rendus contre les romans de femmes, mais que l'on trouve surtout dans ses accusations contre le philosophe irlandais, son style et ses arguments ${ }^{73}$. Publié vingt-huit jours après l'œuvre de Burke ${ }^{74}$ (initialement de façon anonyme et trois semaines après avec le nom de Wollstonecraft), l'ouvrage s'ouvre avec cet avertissement :

Les Reflections on the French Revolution ont d'abord attiré mon attention comme le sujet éphémère du moment; et les lisant davantage pour mon amusement que mon édification, mon indignation a été soulevée par les arguments sophistiques, qui me fâchaient à chaque instant, sous la forme douteuse des sentiments naturels et du sens commun. De nombreuses pages de la lettre qui suit sont le produit des effusions du moment; mais, gonflant imperceptiblement jusqu'à une taille considérable, l'idée me fut suggérée de publier une courte défense de Rights of $\mathrm{Men}^{75}$.

Wollstonecraft entend démentir les principes sur lesquels Burke avait construit son attaque contre la Révolution: dans un style qui, bien que durement critiqué par l'historiographie du siècle dernier, reprend celui défendu par Burke dans Enquiry into the Sublime and Beautiful, la philosophe anglaise affirme qu'elle veut « vous montrer [à Burke], à vous-même, dénudé des magnifiques drapées dans lesquels vous avez enveloppé vos principes tyranniques $»^{76}$.

Si Burke fonde sa défense de l'aristocratie, de la monarchie et de l'Église sur le poids d'une tradition longue de plusieurs siècles, Wollstonecraft condamne cette tradition et ce passé dans lesquels les droits de l'homme n'étaient pas respectés :

Devons-nous rechercher les droits des hommes dans les âges où seuls quelques marques constituaient la seule punition imposée en réparation de la vie d'un homme, et la mort lorsque la propriété des riches était menacée ? Lorsque - je rougis devant la découverte de la dépravation de notre nature - lorsqu'un chevreuil était tué ! Sont-ce là les lois qu'il est naturel d'aimer et sacrilège d'attaquer? Les droits des hommes étaient-ils compris lorsque la loi autorisait et tolérait le meurtre ?77 
Wollstonecraft ne se contente pas d'accuser Burke de se contredire, lui qui avait initialement défendu la Révolution américaine et le Regency Bill; par provocation, elle lui demande s'il ne devrait pas plus justement se définir comme «le champion de la propriété, l'adorateur du veau d'or que le pouvoir a érigé ", plutôt que comme " un ami de la liberté ${ }^{78}$. Mais Wollstonecraft attaque aussi la Constitution anglaise dans laquelle la propriété vaut plus que la liberté. En décrivant les conséquences négatives sur la société anglaise de cette défense de la propriété (parmi lesquelles les mariages précoces et la primogéniture), elle conclut que « la seule sûreté quant à la propriété qu'autorise la nature et que la raison sanctionne est le droit qu'un homme a de jouir des acquisitions que ses talents et son industrie lui ont valu ; et de les transmettre à qui il choisit $"$ ". Quant à la description de la Révolution, elle accuse Burke de ne pas avoir donné une représentation authentique des événements, d'avoir par exemple exagéré «les horreurs du 6 octobre " parce que, intéressé seulement par le sort du roi et de la reine, il n'avait pas compris les soucis des femmes qui doivent travailler pour vivre et qu'il avait indûment définies comme "les abominations inarticulées des furies de l'enfer, sous la forme mensongère des plus viles des femmes" [the unutterable abominations of the furies of hell, in the abused shape of the vilest of women] ${ }^{80}$. Finalement, Wollstonecraft estime insensée l'affirmation de Burke selon laquelle les Français auraient dû considérer l'exemple de la Constitution anglaise et démolit l'entière construction idéologique de Burke en concluant que s'il avait été un citoyen français, il aurait été un révolutionnaire et il aurait tenu les mêmes opinions qu'il avait défendues au cours la guerre d'Indépendance américaine.

A Vindication of the Rights of Men provoqua une controverse, surtout après la publication de la deuxième édition qui portait sur la page de titre le nom de Mary Wollstonecraft. Si l'Analytical Review apprécia l'œuvre de sa collaboratrice et ironisa sur la déception qu'un « chevalier chevaleresque » (chivalrous knight) comme Burke avait dû éprouver en découvrant que "deux de ses adversaires les plus téméraires sont des femmes ${ }^{81}$ [Mary Wollstonecraft et Catherine Macaulay], la Critical Review s'étonnait que "ce défenseur des Droits de l'Homme [était] une Femme $»^{82}$, alors que le Gentleman's Magazine remarquait avec une certaine ironie que «les droits de la femme sont le thème approprié au sexe féminin $»^{83}$.

\section{La Révolution fait-elle des femmes des étrangères à la Cité républicaine?}

En 1792, Mary Wollstonecraft satisfait le Gentleman's Magazine, qui lui avait reproché d'avoir écrit une défense des droits des hommes, en publiant $A$ Vindication of the Rights of Woman ${ }^{84}$. Le texte qui a assuré à Wollstonecraft à partir de la deuxième moitié du dixneuvième siècle la réputation de "première féministe » et qui reste aujourd'hui l'une des œuvres les plus connues et étudiées de la fin du dix-huitième siècle ${ }^{85}$, permet de réfléchir au thème de l'histoire de la Révolution et des femmes, en ouvrant le débat sur l'apport émancipateur ou misogyne de la législation révolutionnaire. Wollstonecraft, en effet, dédie son texte à Charles Maurice de Talleyrand-Périgord en réponse à son Rapport sur l'instruction publique, fait au nom du Comité de constitution ${ }^{86}$, en lui demandant de « reconsidérer ce sujet, et de peser avec maturité ce que j'ai avancé concernant les droits de la femme et d'une éducation nationale $\aleph^{87}$. Wollstonecraft, convaincue du lien profond entre éducation et réforme de la société, avertit Talleyrand et les 
révolutionnaires que la Révolution est destinée à échouer si les femmes ne sont pas associées aux hommes dans une nouvelle éducation nationale.

Défendant les droits des femmes, mon principal argument est fondé sur ce principe simple que si elle n'est pas préparée par son éducation à devenir le compagnon de l'homme, elle arrêtera le progrès de la connaissance et de la vertu ; car la vérité doit être commune à tous, ou elle sera inefficace en ce qui concerne son influence dans la pratique courante. Et comment une femme peut-elle coopérer à moins qu'elle ne sache pourquoi elle devrait être vertueuse ? À moins que la liberté ne renforce sa raison jusqu'à ce qu'elle comprenne son devoir, et voie de quelle manière elle est liée à son propre bien? Si les enfants doivent être éduqués pour comprendre le véritable principe du patriotisme, leur mère doit être une patriote; et l'amour de l'humanité, duquel jaillit le convoi ordonné des vertus, ne peut être produit qu'en prenant en compte l'intérêt moral et civique de l'humanité ; mais l'éducation et la situation de la femme, présentement, lui interdisent de telles investigations ${ }^{88}$.

Wollstonecraft conteste que "l'exclusion des emplois public prononcée contre les femmes soit pour les deux sexes un moyen d'augmenter la somme de leur bonheur mutuel $\aleph^{89}$ et assure que cette exclusion, en plus d'être moralement injuste et contradictoire, met en danger la nouvelle organisation politique et le bonheur collectif puisque «si les femmes n'ont pas l'autorisation de jouir des droits légitimes, elles rendront à la fois les hommes et elles-mêmes vicieux, afin d'obtenir des privilèges illicites $»^{90}$.

À partir de sa propre expérience professionnelle et de l'étude de l'histoire, Wollstonecraft dénonce le système éducatif pour avoir créé de profondes différences entre hommes et femmes et être à l'origine de la faiblesse et du malheur de ces dernières ${ }^{91}$. Un système éducatif fallacieux, pensé par des hommes soucieux de faire des femmes des êtres séduisants et non des épouses fidèles et des mères raisonnables, a fait que « les femmes civilisées du présent siècle, à de rares exceptions, sont uniquement désireuses d'inspirer l'amour, alors qu'elles devraient chérir une ambition plus noble et, par leurs capacités et leurs vertus, susciter le respect $»^{92}$. Wollstonecraft attaque « les tyrans et les sensualistes » qui veulent maintenir les femmes dans l'ignorance : les premiers veulent des esclaves, tandis que les deuxièmes veulent un «jouet " [a plaything $]^{93}$. En particulier, Wollstonecraft s'oppose à Rousseau et à l'éducation réservée à Sophie, dont le seul objectif est celui de rendre la femme agréable et plaisante. Laissant de côté les arguments philosophiques qui démontrent clairement, selon Wollstonecraft, l'immoralité d'un système éducatif négligeant délibérément la raison et les vertus de la moitié des membres de la société, la philosophe s'adresse aux hommes pour démontrer que cette éducation fragilise l'ensemble de l'organisation sociale du fait de l'institution du mariage, étant donné «que leur premier devoir est à elles-mêmes en tant que créatures rationnelles, et le second, par ordre d'importance, en tant que citoyennes, est celui de mère, qui en inclut tant d'autres ${ }^{94}$. Elle nie que des femmes passives et indolentes puissent être des bonnes épouses ou, encore plus rarement, des bonnes mères. En combattant une société qui ne met pas les femmes dans la condition de cultiver les vertus, Wollstonecraft demande une éducation nationale pour toutes les femmes et pas seulement pour un petit nombre de femmes illustres. Dans le chapitre XII, intitulé On national education, Wollstonecraft illustre les avantages d'une éducation nationale commune aux filles et aux garçons, qui permettrait une amélioration générale de la société. Elle conclut son exposé en affirmant qu'

en faisant valoir les droits que les femmes en commun avec les hommes devraient défendre, je n'ai pas tenté d'atténuer leurs fautes, mais de démontrer qu'elles 
étaient la conséquence naturelle de leur éducation et de leur position dans la société. S'il en est ainsi, il est raisonnable de supposer qu'elles changeront leur caractère, et corrigeront leurs vices et leurs folies, lorsqu'elles seront autorisées à être libres dans un sens physique, moral et civique ${ }^{95}$. dans l'Analytical Review ${ }^{97}$, le General Magazine ${ }^{98}$ et le Literary Magazine ${ }^{99}$. La même année, il fut publié aux États Unis ${ }^{100}$ et traduit en français ${ }^{101}$. Il est intéressant de noter que Wollstonecraft avait conclu sa dédicace à Talleyrand en soulignant le désir de faire des observations en France pour confirmer ses principes. Bien qu'elle n'ait pas tenu sa promesse de publier un deuxième volume de son Vindication of the Rights of Woman, on peut néanmoins réfléchir à son interprétation de la Révolution française par rapport aux femmes en analysant An Historical and Moral View of the Origin and Progress of the French Revolution and the Effect it Has Produced in Europe ${ }^{102}$, qu'elle écrivit pendant son séjour en France.

31 Dans ce dernier, Wollstonecraft pose le problème de savoir comment concilier sa défense enthousiaste de la Révolution avec le thème de la violence et de la terreur révolutionnaire ; c'est peut-être pour cette raison qu'elle arrête sa narration à la fin de l'année 1789, sur le modèle de Reflections on the Revolution in France par Burke, et ne décrit pas ce qu'elle voit de ses propres yeux. Dans cette histoire de la Révolution, Wollstonecraft ne néglige pas le rôle des femmes : déjà dans A Vindication of the Rights of Men, elle avait défendu les femmes de la Halle contre les insultes de Burke et elle avait au contraire vivement attaqué Marie-Antoinette, gagnant ainsi d'être appelée la « hyène en jupons » par Horace Walpole ${ }^{103}$. Si, dans sa première attaque contre Burke, elle avait fermement critiqué l'idée des femmes consacrées seulement à la beauté et à l'amour, mais éloignées de la raison, dans An Historical and Moral View of the Origin and Progress of the French Revolution, elle souligne le rôle du gouvernement qui doit défendre une égalité qui n'existe pas dans la nature: "la nature ayant rendu les hommes inégaux, en donnant à certains davantage de pouvoirs physiques et mentaux, le but d'un gouvernement devrait être de détruire cette inégalité en protégeant les faibles $»^{104}$.

Wollstonecraft assure que la Révolution française n'a pas été produite par les machinations ou les intrigues de quelqu'un, pas plus qu'elle n'a été l'effet immédiat d'un bref enthousiasme, mais bien la conséquence naturelle du progrès intellectuel qui touche toutes les communautés ${ }^{105}$. À ce propos, elle trace une brève histoire de la France pour montrer l'évolution et la diffusion de cet esprit, sans oublier, naturellement, le rôle joué par le reste du monde et, en particulier, l'exemple de la Révolution américaine. D'ailleurs, elle souligne que l'histoire de l'Europe montre un progrès général de sorte que «les gouvernements les plus arbitraires en Europe, la Russie exceptée, commencent à traiter leurs sujets comme des êtres humains, dotés de sentiments humains et de certaines capacités de pensée $»^{106}$ et elle définit la liberté comme étant « le droit naturel et imprescriptible de l'homme sans la jouissance duquel il lui est impossible de devenir un être raisonnable ou digne $»^{107}$.

Dans la description des événements de France, Wollstonecraft exalte la prise de la Bastille comme «l'effort le plus incroyable d'un peuple indigné qui ressentit, pour la première fois, qu'il était souverain, et que son pouvoir n'était l'égal que de sa volonté $»^{108}$. Elle précise d'ailleurs que les femmes participent à cette journée " avec autant d'esprit » [equally animated] que les hommes ${ }^{109}$. De plus, Wollstonecraft souligne que les femmes françaises «n'ont pas ces manières factieuses, dédaigneuses si 
répandues chez les Anglaises et, agissant plus librement, elles ont un caractère plus déterminé et, même, plus de générosité $\aleph^{110}$; elle loue, à ce propos, les dons patriotiques de bijoux à l'Assemblée nationale. Toutefois, en présentant les journées des 5 et 6 octobre 1789, la philosophe anglaise introduit une réflexion significative sur le rôle des femmes dans la Révolution française :

Du fait de la jouissance de davantage de liberté que les femmes des autres régions du monde, les femmes de France ont acquis davantage d'indépendance d'esprit que les autres; il a donc été souvent l'objet d'hommes comploteurs depuis la révolution cherchant à se servir d'elles comme d'une sorte de protection, les incitant à commettre quelque acte désespéré, le désignant alors comme une folie, car uniquement provoquée par la rage des femmes, qui n'étaient supposément qu'animées par leurs seules émotions du moment. Ainsi, tôt le 5 octobre, une multitude de femmes furent assemblées par quelque impulsion et, se hâtant à l'hôtel de ville, obligèrent toutes les femmes qu'elles croisaient à les accompagner, pénétrant même dans de nombreuses maisons pour les forcer à suivre leur procession $^{111}$.

$\mathrm{Au}$ contraire de ce qu'elle avait écrit contre Burke dans A Vindication of the Rights of Men, dans ce cas Wollstonecraft définit les femmes de la marche sur Versailles comme étant " pour la plupart des femmes du marché, et les plus vils déchets des rues, des femmes qui avaient rejeté les vertus d'un sexe, sans avoir la capacité d'endosser autre chose que les vices de l'autre ${ }^{112}$. Elle nie par ailleurs qu'il s'agisse d'une manifestation spontanée, qui ne doit pas être confondue avec les multitudes honnêtes qui ont pris la Bastille ${ }^{113}$, mais plutôt le résultat d'une machination orchestrée par le duc d'Orléans. Elle ajoute également que «le fait qu'un corps de femmes puisse se mettre en branle pour demander du secours au roi, ou pour faire des remontrances à l'assemblée en ce qui concerne leur lenteur dans l'élaboration de la constitution est hautement improbable $»^{114}$.

35 À ce dénigrement de l'action féminine doit être enfin ajoutée l'utilisation du mot « efféminé » avec une connotation clairement négative que Wollstonecraft emploie en deux circonstances dans cette description de la Révolution. Bien qu'il s'agisse certes de la signification communément acceptée dans l'anglais (ainsi que dans le français) du dix-huitième siècle, il est quand même intéressant de réfléchir à cette construction qu'elle utilise tout d'abord pour décrire la rapidité des changements révolutionnaires en France :

Ces transitions soudaines d'un extrême à l'autre, qui ne laissent aucune solide conviction, soit pour confirmer soit pour éradiquer la méfiance corrosive, n'étaient nulle part ailleurs qu'à Paris aussi visibles du fait que plusieurs facteurs ont tant et si bien efféminé la raison que les Français peuvent considérés comme une nation de femmes ${ }^{115}$.

Puis, quand elle explique l'explosion de la violence révolutionnaire de la Terreur :

La liberté est un bien précieux qu'il convient de traiter avec révérence et respect. Mais tandis qu'une race efféminée de héros cherche à s'attirer ses sourires avec toutes les délicatesses de la galanterie, c'est à sa postérité plus vigoureuse et naturelle qu'elle se livrera avec toute la brillance modérée des charmes sans artifice $^{116}$.

Il s'agit d'ailleurs d'un élément qu'on retrouve dans d'autres œuvres de Wollstonecraft, surtout en rapport avec l'adjectif masculin : quand elle recensait le premier volume des Letters from France par Williams pour l'Analytical Review, elle y voyait un exemple d'écriture "véritablement féminine " ${ }^{117}$ [truly feminine], tandis qu'en louant l'œuvre de Catherine Macaulay, qu'elle admire beaucoup, elle écrivait que « cet écrivain masculin 
et fervent a dévoué les capacités tout à fait supérieures de son esprit à l'analyse d'un sujet qui, peut-être, inclut un cercle beaucoup plus large d'opinions incertaines $»^{118}$. Dans A Vindication of the Righs of Woman, Wollstonecraft explique ce qu'elle vise derrière cet éloge du masculin : elle espère que les femmes deviendront de jour en jour « de plus en plus masculin " entendant par-là "l'imitation des vertus masculine ou, à plus proprement parler, l'accomplissement de ces talents et de ces vertus dont l'exercice anoblit le caractère humain et qui élève les femmes dans l'échelle des êtres animaux jusqu'à ce qu'elles soient dans la complétude du terme d'humanité $»^{119}$. Derrière cette interprétation dévalorisante du féminin, on trouve certainement la signification dixhuitièmiste du mot, qui d'ailleurs se retrouve aussi dans le français, mais on peut également apercevoir les traces d'une vision typiquement anglaise de la masculinité et de la féminité, jugeant les Français efféminés. Comme l'a souligné Linda Colley, à partir de la deuxième moitié du dix-huitième siècle, les Anglais envisageaient une rivalité intense entre leur culture considérée essentiellement masculine - franche, directe, rationnelle - et celle française, réputée essentiellement efféminée - subtile, sournoise et constamment occupée par la mode, la cuisine, l'étiquette et le sexe ${ }^{120}$.

An Historical and Moral View of the Origin and Progress of the French Revolution, qui aurait dû être le premier tome d'une histoire en plusieurs volumes de la Révolution française, a été bien accueilli par le public anglais, même s'il n'était pas entièrement en adéquation avec le climat national: le volume a été critiqué surtout au sujet de la reine que Wollstonecraft avait qualifiée de " dupe des fripons qu'elle a favorisés $~{ }^{121}$ dans l'affaire du collier et peinte comme une habile dissimulatrice qui avait passé toute son existence à s'occuper d'affaires totalement inutiles et puériles.

Dans les Letters from France d'Helen Maria Williams, les femmes jouent au contraire un rôle de premier plan. Dès les premières lettres, Williams évoque le rôle des Françaises dans l'histoire de la Révolution : elles participent à la prise de la Bastille, à la Fête de la Fédération, mais c'est surtout dans le cinquième volume (celui qui couvre les événements entre le 31 mai et le 10 thermidor) que le rôle principal des femmes émerge. Williams ouvre son texte avec cette description : "c'est à la première de ces époques que l'on a vu un ignoble et sanguinaire despote s'asseoir sur le débris d'un trône : la seconde a renversé le tyran et rendu la vie à la liberté, encore toute sanglante des blessures dont elle étoit couverte $»^{122}$. La liberté, représentée métaphoriquement comme une femme blessée et saignante, reproduit une figure typique de la rhétorique post-thermidorienne de Williams. Selon Deborah Kennedy, l'image de la Liberté en tant que "femme sensible, blessée par les abus des Jacobins " [woman of sensibility, wounded by the ill-usage of the Jacobins ${ }^{123}$ est très représentative du style post-thermidorien, puisqu'elle contraste avec la représentation de la Liberté comme Hercule viril voulu par les Jacobins et bien étudiée par Lynn Hunt ${ }^{124}$.

Cependant, on peut considérer que la représentation de la Liberté en tant que femme couverte de sang est particulièrement significative en vertu du rôle que les femmes jouent dans le récit de Williams pendant la période de la Terreur. Analysant la production littéraire entre l'an II et Thermidor, ce sont précisément les personnages féminins qui subissent une transformation radicale de mères héroïques prêtes à se sacrifier pour leur patrie à victimes sans défense de violences brutales. En décrivant l'exécution d'une jeune mère dans un village du nord de la France, Williams, dans le troisième volume de ses lettres (jamais traduites en français), abandonne sa réserve habituelle et écrit que « lorsqu'elle reçut le coup fatal, les ruisseaux du nourrissement 
maternel jaillirent rapidement de son sein et, se mêlant avec son sang, baignèrent son bourreau ${ }^{125}$. De façon paroxystique, la condamnation à mort d'une femme qui allaitait son bébé rappelle, mais, en même temps, déconsacre l'image de la république comme mère qui nourrit ses enfants. Le blanc du lait et le rouge du sang qui tachent le bourreau en symbolisant la vie et la mort, créent l'une des images les plus indélébiles du règne de la Terreur. D'ailleurs, dans ce volume, on trouve, dans la description du prétendu plan du Comité de salut public « de l'extermination générale des habitants de la Vendée " [of the general extermination of the inhabitants of the Vendée] ${ }^{126}$, l'esquisse dramatique des actes de violences commis contre les femmes comme les soi-disant mariages républicains. Williams décrit d'ailleurs la mort de chagrin d'une jeune fille de quatorze ans qui voit toute sa famille massacrée :

Il emmena cette jeune fille qui rampait faiblement aux pieds de sa mère tandis que ses sœurs, n'étant âgées que de quelques années de plus, étaient condamnées à mourir. Mais mourir ensemble paraissait à présent leur seule consolation; elles refusèrent de se séparer; et une certaine violence fut utilisée pour forcer l'enfant qui, peu de temps après le meurtre de sa famille, mourut d'un cœur brisé ${ }^{127}$.

41 Mais c'est dans le volume traduit en français en 1795 et participant au contexte de la réaction thermidorienne qu'on trouve davantage cette représentation des femmes: dans la peinture du bain de sang de la Terreur, Williams note que " parmi les victimes des tyrans les femmes se sont particulièrement distinguées par leur étonnante fermeté au moment de la mort $»^{128}$. L'insistance sur la représentation du «sang des femmes [qui] couloit tous les jours sur l'échafaud $»^{129}$ sert tout d'abord efficacement à dénoncer les crimes «de ces monstres implacables $»^{130}$, dont la fureur était dirigée, selon Williams, précisément vers ce sexe que l'homme était appelé à protéger. En même temps, l'accent mis sur la fermeté avec laquelle les femmes avaient fait face à la condamnation à mort ouvrait une réflexion sur le comportement politique des Françaises pendant la Révolution, alimentée d'ailleurs par l'opposition manichéenne que Williams établissait entre les adeptes de Robespierre et les femmes sacrifiées pour la liberté. Cette représentation des femmes servait certainement à aligner l'œuvre de Williams sur le climat politique anglais dominé par la répression à la suite des procès pour trahison de 1794 contre le mouvement radical ${ }^{131}$, mais avait surtout la fonction de justifier le soutien de l'auteure à la Révolution et à faire oublier, en vertu de sa proximité avec les femmes sacrifiées à la liberté, sa collaboration au gouvernement révolutionnaire de l'an II via l'imprimerie anglaise de Stone.

Dans la description de la journée du 2 juin 1793, l'auteure insistait sur le rôle joué par Robespierre et par «ses gardes-du-corps femelles, qui s'intituloient la société des femmes révolutionnaires, et qui s'étoient présentées les premières dans les couloirs de la Convention, armées de poignards et de pistolets, dont elles menaçoient les députés qui tentoient de sortir $»^{132}$. Ces femmes, faisait remarquer Williams, s'étaient montrées les plus ardentes auxiliaires des conspirateurs, "elles avoient succédé aux poissardes $»^{133}$ :

ces politiques femelles ouvrirent des assemblées délibérantes, présentèrent leurs vues à la Convention, et influencèrent ses débats, par leurs vociférations dans les tribunes qu'elles occupoient presqu'exclusivement. Dans les journées tumultueuses qui précédèrent le 31 de mai, elles avoient personnellement monté la garde à la Convention ${ }^{134}$. comportement héroïque et sacrificiel de Charlotte Corday et de madame Roland. La 
première, poussée par l'étude de l'histoire ancienne à un «amour très-ardent pour la liberté »"135, avait conçu le meurtre de Marat comme "un devoir dont elle s'étoit acquittée vis-à-vis de son pays et de toute l'humanité, en arrachant la vie à un monstre, dont la doctrine sanguinaire provoquoit la guerre civile et l'anarchie ${ }^{136}$. Williams s'attarde sur la description de la dignité dont cette femme fait preuve lors du procès ( «elle écouta sa sentence avec beaucoup de tranquillité et d'attention »"17), ainsi qu'au moment de son exécution, quand elle monte à l'échafaud avec un pas ferme, déterminée à mourir dignement.

Madame Roland est en revanche décrite comme « une des plus étonnantes femmes que la France ait jamais produites $»^{138}$. Williams avait rencontré la femme du ministre à son arrivée en France et, en décrivant son procès et sa mort, elle trahissait une émotion sincère.

J'allois la voir dans la prison de Sainte Pélagie, où son âme supérieure aux circonstances conservoit toujours la même sérénité [...] Elle me dit qu'elle s'attendoit à périr; et l'air de résignation qu'elle avoit en me parlant, ne me laissa point douter qu'elle ne fut prête à recevoir la mort avec fermeté digne de son grand caractère ${ }^{139}$.

Seul le souvenir de sa fille de treize ans, bientôt orpheline, avait été capable de miner la fermeté de madame Roland. Williams a insisté précisément sur les attributs maternels de la victime pour souligner la monstruosité ${ }^{140}$ de la tyrannie de Robespierre: «le courage de cette infortunée victime de la liberté ne pouvoit résister au souvenir déchirant de son mari et de sa fille; elle ne trouvoit plus dans son cœur sensible d'autre sentiment que celui d'une mère et d'une épouse au désespoir $»^{141}$.

Mais c'est dans Sketches of Manners and Opinions in the French Republic ${ }^{142}$, traduit en français ${ }^{143}$ par Sophie Grandchamp, que Williams exprime nettement son avis sur la Révolution et les femmes ${ }^{144}$. Williams ouvre son texte en se défendant des calomnies et des accusations qui s'abattent toujours contre une femme qui décide de s'occuper de politique :

je connois les critiques qu'on s'est permises sur mon sexe, lorsqu'il a voulu traiter des sujets politiques; et je n'ignore pas les calomnies dirigées contre moi à Londres par des écrivains qui se qualifient d'anti-jacobins; mais quelle que puisse être la malignité de leur but, leurs traits tombent sans causer de blessures dangereuses. Ceux qui ont vécu au milieu des événemens de la révolution française, ont appris à parer, ou à mépriser des coups plus redoutables ${ }^{145}$.

En mettant de côté l'accusation de jacobinisme, puisqu'elle déclare que la terreur est le système politique qu'elle abhorre le plus, Williams espère que, malgré les crimes et les malheurs qui ont souillé la Révolution, son nom sera toujours rappelé « parmi celui des personnes qui essayèrent d'en défendre la cause $»^{146}$. Dans son bilan de la Révolution française, Williams essaye de mettre en perspective ce qu'elle définit comme les "calamités momentanées ${ }^{147}$ : «l'humanité est rentrée dans ses droits, de cruels abus sont détruits, le malheur semble enfin avoir épuisé ses traits ${ }^{148}$. Pour savoir si la Révolution a accru ou diminué le bonheur en France, elle cite « la situation heureuse de l'habitant des campagnes " passé de la pauvreté à une sorte d'opulence, sans oublier " ce que la révolution a fait pour sa laborieuse épouse $»^{149}$.

Mais c'est après avoir décrit la révolution en Suisse et à Naples ${ }^{150}$ que Williams s'interroge sur les conséquences de la Révolution française pour les femmes. Dans la lettre XXV, Williams admet que les femmes françaises se désintéressent de la politique et que, dans la guerre des factions, elles ont maintenu une neutralité parfaite : 
la révolution s'est présentée à leurs yeux comme un événement dont la réussite étoit douteuse, et les suites à craindre; la République qui en a été le résultat a souvent eu un aspect sévère et menaçant, qui a porté l'effroi chez les hommes; pouvons-nous donc être surpris que mon sexe ait repoussé ses embrassemens fraternels ${ }^{151}$ ?

Williams doute que les bienfaits du nouveau gouvernement de Napoléon soient un subside suffisant pour leur faire abandonner cette neutralité : «à la vérité, les femmes peuvent répondre qu'il s'agit moins de savoir si elles ont gagné, que si elles ont obtenu de la révolution tout ce qu'elles devoient en attendre $»^{152}$. Williams précise que les femmes ne demandent pas de former un Sénat à part,

elles ne demandent point à conduire des armées, à régler l'État, à devenir ministres ou directeurs. Cependant, puisque la nation se prêtoit à faire l'expérience de différents modes de gouvernement, elle auroit pu essayer de nos talens en ce genre, et peut-être ne s'en seroit-elle pas plus mal trouvés. Ces femmes pourroient encore vous observer en passant, qu'elles ont porté avec gloire le sceptre de l'empire, et que nommées législatrices, il est douteux, malgré leur penchant à la domination, qu'elles eussent décrété plus de quarante mille de ces lois, dont un grand nombre a été si funeste à la République ${ }^{153}$.

50 En citant les propositions en faveur des femmes écrites par Nicolas de Condorcet, Emmanuel Joseph Sieyès et Charles Guillaume Théremin, Williams, comme Wollstonecraft, condamne le manque d'éducation pour les femmes. Elle espère que bientôt les législateurs sentiront la nécessité «d'établir des écoles publiques où les femmes puissent recevoir une éducation digne d'elles, et des fonctions que la nature et la société leur imposent ${ }^{154}$. Enfin, en commentant le projet de Théremin, qui demande de lier le sort des femmes à la République à travers des commissions sur l'instruction et sur les fêtes nationales, mais nie le droit de vote parce que «la nature a formé les femmes pour être unies à un individu de notre sexe, par conséquent elles doivent avoir les mêmes volontés et les mêmes intérêts ${ }^{155}$, Williams répond avec ce qui peut sembler une provocation voilée, quand elle affirme que

quelque nouvelle Thalestris, prenant les armes pour défendre les droits des femmes, hésiteroit probablement à recevoir cette proposition et ses conséquences. Elle observeroit que le droit politique n'est pas plus aliéné par cette union que par toute autre association civile ${ }^{156}$.

51 Williams conclut son chapitre sur les femmes en admettant que la Constitution de Bonaparte peut avoir un certain attrait pour les femmes ${ }^{157}$ et termine en rappelant l'héroïsme des femmes qui sont montées sur l'échafaud et l'exemple des femmes de Naples qui ont montré une dévotion et un courage sublimes en prouvant " jusqu'à quel point mon sexe peut se dégrader par le vice, et à quelle hauteur il peut s'élever par ses vertus $»^{158}$.

Mary Wollstonecraft et Helen Maria Williams, persuadées de la nécessité de supprimer les privilèges de l'Ancien Régime et de réformer la société, ont vu dans la Révolution française l'aube d'une nouvelle ère. Toutes les deux ont accueilli avec enthousiasme la nouvelle de la prise de la Bastille et l'irruption sur la scène publique du peuple formé par des hommes et des femmes déterminés à garantir à la nation une nouvelle liberté. Toutes les deux ont décidé de s'installer en France pour voir de leurs propres yeux le chemin de la Révolution et prendre part aux changements politiques, sociaux et culturels qui, de la France, auraient ensuite touché l'Europe entière. Toutes les deux ont vu avec frayeur l'explosion de la violence révolutionnaire en l'an II et les persécutions contre les Anglais: Williams n'a d'ailleurs pas réussi à fuir le décret 
d'arrestation. Enfin, toutes les deux ont été déçues par le sort des femmes en révolution: Mary Wollstonecraft, qui avait souhaité voir la mise en place d'une éducation nationale pour les deux sexes ensemble afin d'assurer la formation de bons citoyens, a vu avec regret l'établissement d'un système d'éducation qui laissait les femmes de côté, tandis que Helen Maria Williams a reproché aux révolutionnaires leur manque de considération pour les talents, les capacités et les idées des citoyennes, alors même que la nation s'apprêtait à faire l'expérience de différents modes de gouvernement. Femmes et étrangères sur le sol français, toutes les deux ont dénoncé les limites d'une révolution qui considérait les femmes étrangères au corps politique de la nation régénérée.

Évidemment, les itinéraires biographiques de Wollstonecraft et Williams, avec leur mobilité et leur prise de parole publique, représentent d'une certaine façon des cas exceptionnels dans l'Europe de la fin du dix-huitième siècle. Toutefois, on ne doit pas oublier le rôle majeur joué par leurs œuvres dans la discussion et l'interprétation de la Révolution : elles s'inscrivent dans un débat international sur la Révolution et jouent tout d'abord un rôle de médiation culturelle entre l'Angleterre et la France ${ }^{159}$. Dans l'affrontement des positions pour ou contre la Révolution française que connaît le monde britannique dès 1789 , la réflexion politique théorique ne doit pas obscurcir un riche débat fait de littérature fictionnelle dans lequel plusieurs femmes s'inscrivent en s'adressant aux hommes et femmes qui dans les romans ne cherchent pas seulement une évasion de la vie quotidienne. Comme Hannah More critiquait les événements de France dans son best-seller Village politics ${ }^{160}$, ainsi Mary Wollstonecraft, Helen Maria Williams, Charlotte Turner Smith, Mary Hays défendaient dans la Révolution une opportunité de changement de la société, dans laquelle enfin les femmes auraient pu jouer un rôle non subordonné à celui des hommes.

\section{NOTES}

1. Sur ces aspects, voir la récente synthèse de Harry T. DICKINSON, Pascal DUPUY, Le temps des cannibales. La Révolution française vue des îles britanniques, Paris, Vendémiaire, 2019.

2. Richard PRICE, A Discourse on the Love of Our Country, Delivered on Nov. 4, 1789, at the Meeting-House in the Old Jewry, to the Society for Commemorating the Revolution in Britain, Londres, E. Powars, 1789.

3. Edmund BURKE, Reflections on the Revolution in France and on the Proceedings in Certain Societies in London Relative to that Event. In a Letter Intended to Have Been Sent to a Gentleman in Paris, Londres, Dodsley, 1790.

4. Mary wollstonecraft, Original Stories from Real Life with Conversations, Calculated to Regulate the Affections and Form the Mind to Truth and Goodness, Londres, Johnson, 1788, p. 28: «I told you, Robin was confined in a gaol. In France they have a dreadful one. The poor wretches who are confined in it live entirely alone; have not the pleasure of seeing men or animals; nor are they allowed books. They live in comfortless solitude [...] One miserable captive found a spider, he nourished it for two or three years; it grew tame and partook of his lonely meal. His keeper observed it, and mentioned the circumstance to a superior, who ordered him to crush it. In vain did the man beg to have his spider spared ». 
5. Alan RICHARDSON, «Mary Wollstonecraft on Education », dans Claudia L. Johnson (ed.), The Cambridge Companion to Mary Wollstonecraft, Cambridge, Cambridge University Press, 2002, p. 24-41.

6. Mary WOLLSTONECRAFT, Thoughts on the Education of Daughters, Londres, Johnson, 1787.

7. Stéphanie Félicité du Crest, comtesse de GENLIS, Adèle et Théodore ou Lettres sur l'éducation contenant tous les principes relatifs à l'éducation des princes, des jeunes personnes et des hommes, Paris, Lambert \& F. J. Baudouin, 1782.

8. Mary WOLLSTONECRAFT, Erzählungen für Kinder, aus dem Englischen übersetzt, Schnepfenthal, im Verlage der Buchhandlung der Erziehungsanstalt, 1795.

9. ID., Marie et Caroline, ou Entretiens d'une institutrice avec ses élèves, orné de cinq gravures, traduit en français par A. J.N. Lallemant, Paris, Dentu, 1799.

10. John LOCKE, Some Thoughts Concerning Education, Londres, printed for A. and J Churchill, 1693.

11. Jean-Jacques RouSSEAU, Émile ou De l'éducation, La Haye, Chez Jean Néalme, 1762.

12. Pour une réflexion sur la théorie de l'éducation de Mary Wollstonecraft, voir Morwenna GRIFFITHS, «Educational Relationships: Rousseau, Wollstonecraft and Social Justice », Journal of Philosophy of Education, 48, 2, 2014, p. 339-354 et Elizabeth FRAZER, "Mary Wollstonecraft and Catharine Macaulay on Education ", Oxford Review of Education, 37, 5, 2011, p. 603-617.

13. Janet TODD, Mary Wollstonecraft. A Revolutionary Life, Londres, Bloomsbury Reader, 2014.

14. Letter to Everina Wollstonecraft (n.67), dans Ralph M. Wardle (ed.), Collected Letters of Mary Wollstonecraft, Londres, Cornell University Press, 1979, p. 164 : «Mr. Johnson, whose uncommon kindness, I believe, has saved me from despair [...] assures me that if I exert my talents in writing I may support myself in a comfortable way. I am then going to be the first of a new genus ».

15. Mitzi MYERS, « Child's Play as Woman's Peace Work: Maria Edgeworth's 'The Cherry Orchard' Historical Rebellion Narratives, and Contemporary Cultural Studies », dans Beverly Lyon Clark \& Margaret R. Higonnet (ed.), Girls, Boys, Books, Toys: Gender in Children's Literature and Culture, Baltimore, Johns Hopkins University Press, 1999.

16. Mary wollstoneCRAFt, Mary: A Fiction, Londres, Johnson, 1788. Sur les romans de Wollstonecraft, voir Christine HIVET, Voix de femmes. Roman féminin et condition féminine de Mary Wollstonecraft à Mary Shelley, Paris, Pens, 1997.

17. On peut citer, à titre d'exemple, l'introduction au compte-rendu de Edward and Harriet, or the Happy Recovery: a sentimental Novel by a lady, cité dans Janet Todd \& Marilyn Butler (ed.), The Works of Mary Wollstonecraft, vol. 7, Londres, William Pickering, 1989, p. 19 : « The Happy Recovery is an heterogeneous mass of folly, affectation and improbability. Metaphors and vulgarism abound ».

18. Jacques NECKER, De l'importance des opinions religieuses, Londres, Panckoucke, 1788.

19. Richard PRICE, A Discourse on the Love of Our Country, op. cit.

20. Mary WOLLSTONECRAFT, « Compte-rendu de Richard Price, A Discours on the Love of Our Country ", dans Janet Todd \& Marilyn Butler (ed.), The Works of Mary Wollstonecraft, op. cit, vol.7, p. 185 : «love, the result of the reason, not the undirected impulse of nature, ever tending to selfish extremes».

21. Ibid. : «the light [that all you friends of freedom] have struck out, after setting AMERICA free, reflected to FRANCE, and there kindled into a blaze that lays despotism in ashes, and warms and illuminates EUROPE ».

22. Helen Maria WiLliams, Julia. A Novel. Interspersed with Some Poetical Pieces, 2 vol., Londres, T. Cadell, 1790.

23. Lionel D. WOODWARD, Une Anglaise amie de la Révolution française: Hélène-Maria Williams et ses amis, Paris, Honoré Champion, 1930; Deborah KENNEDY, Helen Maria Williams and the Age of Revolution, Londres, Bucknell University Press, 2002.

24. Helen Maria Williams, A Poem on the Bill Lately Passed for Regulating the Slave Trade, Londres, T. Cadell, 1788. 
25. ID., Souvenirs de la Révolution française, traduit par C. C. [Charles Coquerel], Dondey-Dupré, Paris, 1827 , p. 197 : «I distributed, on my first visit to Paris, several engravings of the plan of the slave ships, and several of the small Wedgwood cameos, which represent the black man on his knees, pleading his cause and showing his chains $»$.

26. ID, Julia... op. cit.

27. Jean-Jacques RousSEAU, Julie, ou la Nouvelle Héloïse, Amsterdam, chez Marc-Michel Rey, 1761.

28. Helen Maria williams, The Bastille. A Vision (vv.71-72), dans Julia..., op. cit., vol. 2, p. 221.

29. Sur le discours radical anglais et sur la tension constitutive qui imprègne la conception de l'identité britannique et les rapports avec les révolutionnaires américains et français, voir Rémy DUTHILLE, Le discours radical en Grande-Bretagne, 1768-1789, Oxford, Voltaire Foundation, 2017.

30. Deborah KENNEDY, Helen Maria Williams and the Age of Revolution..., op. cit., p. 53.

31. Richard PRICE, A Discourse on the Love of Our Country..., op. cit.

32. Helen Maria WILLIAMS, Letters Written in France, in the Summer of 1790, to a Friend in England. Containing Various Anecdotes Relative to the French Revolution; and Memoirs of Mons. and Madame du F..., Londres, T. Cadell, 1790 ; ID., Letters from France. Containing Many New Anecdotes Relative to the French Revolution, and the Present State of French Manners, Londres, G. \& J. Robinson, 1792. ID., Letters from France; Containing a Great Variety of Interesting and Original Information concerning the Most Important Events That Have Lately Occurred in that Country in the Years 1790, 1791, 1792, and 1793, and Particularly Respecting the Campaign of 1792, 2 vol., Dublin, J. Chambers, 1794. ID., Letters Containing a Sketch of the Politics of France, From the Thirty-first of May 1793, till the Twenty-eighth of July 1794, and of the Scenes Which Have Passed in the Prisons of Paris, 2 vol., Londres, G.-G. et J. Robinson, 1795. ID., Letters Containing a Sketch of the Scenes Which Passed in Various Departments of France During the Tyranny of Robespierre, and of the Events Which Took Place in Paris on the 28th of July 1794, Londres, G.G. et J. Robinson, 1795.

ID., Letters Containing a Sketch of the Politics of France, From the Twenty-Eighth of July 1794, to the Establishment of the Constitution of 1795, and of the Scenes Which Have Passed in the Prisons of Paris, Londres, G.-G. et J. Robinson, 1796.

33. Helen Maria williams, Les lettres écrites en France à une amie en Angleterre pendant l'année 1790, Contenant l'histoire des Malheurs de M. du F***, Paris, imprimerie de Garnery, libraire, rue Serpente, n. $17,1791$.

34. Ibid., p. 74.

35. Ibid., p. 1.

36. Ibid., p. 10.

37. Ibid., p. 23.

38. Ibid., p. 45.

39. Ibid., p. 51-52. L'engagement d'Helen Maria Williams en faveur de l'émancipation des Noirs est durable. Il ne faut pas oublier que Williams joua un rôle central dans la seconde Société des Amis des Noirs et des Colonies, rétablie entre l'automne 1797 et le printemps 1799. Voir Marcel DORIGNY, Bernard GAINOT, La Société des Amis des noirs, 1788-1799, Paris, Éditions Unesco, 1998, p. 299-396.

40. Helen Maria WiLliams, Les lettres écrites en France à une amie en Angleterre pendant l'année 1790, op. cit., p. 86.

41. Ibid., p. 173.

42. Ibid., p. 178.

43. Ibid., p. 71.

44. Ibid., p. 74 .

45. ID., Letters from France. Containing Many New Anecdotes Relative to the French Revolution, op. cit., p. 205 : «the genuine principles of enlightened freedom will soon be better comprehended, and 
may perhaps at no distant period be adopted by all the nations of Europe. Liberty may bring 'her sons from afar, and her daughters from the ends of the earth' ".

46. ID., Lettres sur les événemens qui se sont passés en France, depuis le 31 mai 1793 jusqu'au 10 thermidor, Paris, imprimerie de la rue de Vaugirard, n. 970, [1795].

47. Barbaroux, Birotteau, Brissot, Buzot, Chambon, Gensonné, Gorsas, Grangeneuve, Guadet, Lanjuinais, Lasource, Lehardy, Lesage, Lidon, Louvet, Pétion, Salle, Valazé, Vergniaud et dix membres de la commission des Douze.

48. Ibid., p. 13.

49. Ibid., p. 58.

50. Ibid., p. 36.

51. Michel BIARD, La liberté ou la mort. Mourir en député, 1792-1795, Paris, Tallandier, 2015 ; Harold T. PARKER, The Cult of Antiquity and the French Revolutionaries: A Study in the Development of the Revolutionary Spirit, New York, Octagon, 1965 ; Dorinda OUTRAM, The Body and The French Revolution: Sex, Class, and Political Culture, New Haven, Yale University Press, 1989.

52. Helen Maria WILLIAMs, Lettres sur les événemens qui se sont passés en France, op. cit., p. 125.

53. Ibid., p. 94.

54. Ibid., p. 130.

55. Hervé Leuwers a souligné que cette attention portée au parcours personnel de Robespierre, présente depuis la plus ancienne historiographie (et encore observable dans les travaux plus récents), découle d'une dimension originale et peu étudiée des discours du député qui est la «tentation de l'autobiographie» (Hervé LEUWERS, «Aux origines du 'problème' Robespierre: l'historien face à ses interrogations ", H-France Salon, vol. 7, nº 15, 2015, p. 1-10).

56. Lievain-Bonaventure PROYART, La vie et les crimes de Robespierre, surnommé le tyran, depuis sa naissance jusqu'à sa mort, Augsbourg, Chez tous les libraires, 1795.

57. Helen Maria WiLliams, Lettres sur les événemens qui se sont passés en France, op. cit., p. 183.

58. Ibid., p. 134.

59. Helen Maria williams, A Tour in Switzerland; or, A View of the Present State of the Government and Manners of those Cantons: with Comparative Sketches of the Present State of Paris, 2 vol., Londres, G. G. et J. Robinson, 1798.

60. ID., Nouveau voyage en Suisse, contenant une peinture de ce pays, de ses mœurs et de ses gouvernements actuels, avec quelques traits de comparaison entre les usages de la Suisse et ceux de Paris moderne, trad. par J. B. Say, 2 vol., Paris, Charles Pougens, 1798. Pour une analyse ponctuelle de l'ouvrage, voir Bernard GAINOT, «La République et la pastorale. Autour du Nouveau voyage en Suisse de Helen Maria Williams (1798)», dans Pierre Serna \& Gilles Bertrand (dir.), La République en voyage, Rennes, Presses universitaires de Rennes, 2013, p. 265-281.

61. Pour approfondir la relation entre Williams et Stone, voir John G. ALGER, Englishmen in the French Revolution, Londres, Sampson Low, 1889 et Madeleine B. STERN, « The English Press in Paris and Its Successors, 1793-1852», The Papers of the Bibliographical Society of America, 74, 4, 1980, p. 307-359. Sur les journaux anglais dans la France révolutionnaire, voir Simon MACDONALD, «English-Language Newspapers in Revolutionary France », Journal for Eighteenth-Century Studies, 36, I, 2013, p. 17-33.

62. Voir la thèse de doctorat de Sylvie KLEINMAN, "Translation, the French Language and the United Irishmen », Dublin City University, 2005, p. 100-106.

63. Sur le rôle de l'hôtel White pendant la Révolution et les relations de Wollstonecraft et Williams, voir Rachel ROGERS, «Vectors of Revolution: The British Radical Community in Early Republican Paris, 1792-1794 », thèse de doctorat en anglais, université Toulouse le Mirail, 2012 ; ID, «White's Hotel: A Junction of British Radical Culture in Early 1790s Paris », Caliban, n 33, 2013, p. 153-172; Stéphanie GOURDON, «Mary Wollstonecraft et Helen Maria Williams. Étude du 
(nouveau) roman au féminin ", Revue de la Société d'études anglo-américaines des XVII et XVIII ${ }^{e}$ siècles, 2013, p. 143-153.

64. Mathieu FERRADOU, « Histoire d'un 'festin patriotique' à l'hôtel White (18 novembre 1792) : les Irlandais patriotes à Paris, 1789-1795 ", Annales historiques de la Révolution française, $\mathrm{n}^{\circ}$ 382, 2015, p. 123-143 ; John Goldworth ALGER, « The British Colony in Paris, 1792-1793 », The English Historical Review, 1898, p. 672-694.

65. Le Patriote français, 1199, 21 novembre 1792, p. 588.

66. Ibid.

67. Charlotte Turner SMITH, Desmond, Londres, Broadview Press, 1792.

68. ID., Desmond ou l'amant philanthrope, traduit de l'anglais par L.C.D., 4 vol., Paris, Chez Denné, 1793.

69. Mary WOLLSTONECRAFT, A Vindication of the Rights of Men, in a Letter to the Rights Honorable Edmund Burke, Occasioned by his Reflections on the Revolution in France, second edition, Londres, Johnson, 1790. ID., Une Anglaise défend la Révolution française. Réponse à Edmund Burke, traduction et introduction de Marie-Odile Bernez, notes établies par Marie-Odile Bernez et Marcel Dorigny, Paris, CTHS, 2003.

70. Janet TODD, Mary Wollstonecraft. A Revolutionary Life, op. cit.

71. Morning Chronicle, 26 novembre 1792.

72. John Hurford Stone to William Stone [n. d.], dans The Trial of William Stone, Londres, Martha Gurney, 1796, p. 85-86.

73. Sur l'utilisation de la satire comme mode de connaissance historique, voir Nathalie ZIMPFER, "Sense and sensibility: Mary Wollstonecraft as Active Witness to History », Études Épistémè. Revue de littérature et de civilisation (XVI ${ }^{e}$-XVIII ${ }^{e}$ siècles), $\mathrm{n}^{\circ}$ 19, 2011, https://journals.openedition.org/ episteme/633.

74. Randall SESSLER, « Recasting the Revolution: The Media Debate Between Edmund Burke, Mary Wollstonecraft, and Thomas Paine ", European Romantic Review, n² 25, 2014, p. 611-626.

75. Mary WOLLSTONECRAFT, A Vindication of the Rights of Men op. cit., p. III-IV : « Mr Burke's Reflections on the French Revolution first engaged my attention as the transient topic of the day; and reading it more for amusement than information, my indignation was roused by the sophistical arguments, that every moment crossed me, in the questionable shape of natural feelings and common sense. Many pages of the following letter were the effusions of the moment; but, of swelling imperceptibly to a considerable size, the idea was suggested of publishing a short vindication of the Rights of Men ».

76. Ibid., p. 88 : « to shew you [Burke] to yourself, stripped of the gorgeous drapery in which you have enwrapped your tyrannic principles ». Sur le langage de Burke accusé de voiler la vérité de la raison, voir Gillian RUSSELL, The Theatres of War: Performance, Politics, and Society, 1793-1815, Oxford, Clarendon Press, 1995.

77. Ibid., p. 19 : « Are we to seek for the rights of men in the ages when a few marks were the only penalty imposed for the life of a man, and death for death when the property of the rich was touched? When-I blush to discover the depravity of our nature-when a deer was killed! Are these the laws that it is natural to love, and sacrilegious to invade? Were the rights of men understood when the law authorised or tolerated murder $»$.

78. Ibid., p. 20 : «the champion of property, the adorer of the golden image which power has set up " plutôt que comme « a friend of liberty ».

79. Ibid., p. 51 : « the only security of property that nature authorizes and reason sanctions is, the right a man has to enjoy the acquisitions which his talents and industry have acquired; and to bequeath them to whom he chooses $»$.

80. Ibid., p. 67.

81. Analytical Review, VIII, 1790, p. 416 : « that two of the boldest of his adversaries are women ». 
82. Critical Review, 70, 1790, p. 694-696: «this Defender of the Rights of Man [was] a Woman ».

83. The Gentleman's Magazine, $61,1,1791$, p. 151-154: «the rights of woman were the proper theme of the female sex ".

84. Mary wollstonecraft, A Vindication of the Rights of Woman, with Strictures on Political and Moral Subjects, Londres, Johnson, 1792.

85. Enit Karafili STIENER, Called to Civil Existence. Mary Wollstonecraft's A Vindication of the Rights of Woman, Amsterdam, Rodopi, 2014. Karen OFFEN, «Was Mary Wollstonecraft a feminist? A comparative re-reading of A Vindication of the Rights of Woman, 1792-1992 ", dans Karen Offen (ed.), Globalizing Feminisms, 1789-1945, Londres \& New York, Routledge, 2012, p. 5-17 ; Roberta Adelaide MODUGNo, Mary Wollstonecraft. Diritti umani e Rivoluzione francese, Soveria Mannelli, Rubbettino, 2002 ; Craig Calvin MILLER, Mary Wollstonecraft and the Rights of Women, Greensboro, Morgan Reynolds, 1999.

86. Charles Maurice de TALLEYRAND-PÉRIGORD, Rapport sur l'instruction publique, fait au nom du Comité de constitution, à l'Assemblée nationale, les 10, 11 et 19 septembre 1791, Paris, Imprimerie nationale, 1791.

87. Mary Wollstonecraft, A Vindication of the Rights of Woman, op. cit., p. III : « reconsider the subject, and maturely weight what I have advanced respecting the rights of woman and national education ".

88. Ibid., p. VI-VII : "Contending for the rights of woman, my main argument is built on this simple principle, that if she be not prepared by education to become the companion of man, she will stop the progress of knowledge and virtue; for truth must be common to all, or it will be inefficacious with respect to its influence on general practice. And how can woman be expected to cooperate unless she know [sic] why she ought to be virtuous? Unless freedom strengthen her reason till she comprehend [sic] her duty, and see in what manner it is connected with her real good? If children are to be educated to understand the true principle of patriotism, their mother must be a patriot; and the love of mankind, from which an orderly train of virtues spring, can only be produced by considering the moral and civil interest of mankind; but the education and situation of woman, at present, shuts her out from such investigations ».

89. Charles Maurice de TALLEYRAND-PÉRIGORD, Rapport sur l'instruction publique, op. cit., p. 116.

90. Mary WOLlSTONECRAFT, A Vindication of the Rights of Woman, op. cit., p. XIII : « if women are not permitted to enjoy legitimate rights, they will render both men and themselves vicious, to obtain illicit privileges ».

91. Sur le débat sur l'éducation des femmes au cours de la Révolution française, voir Caroline FAYOLLE, La femme nouvelle. Genre, éducation, Révolution (1789-1830), Paris, Éditions du CTHS, 2017 et ID., « Des petites républiques de filles. Projets et expérimentations pédagogiques révolutionnaires (1793-1794) », La Révolution française, $\mathrm{n}^{\circ} 4,2013$, https://doi.org/10.4000/lrf.803.

92. Mary WOllstonecraft, A Vindication of the Rights of Woman, op. cit., p. $2:$ «the civilized women of the present century, with a few exceptions, are only anxious to inspire love, when they ought to cherish a nobler ambition, and by their abilities and virtues exact respect ».

93. Ibid., p. 45.

94. Ibid., p. 331 : «their first duty is to themselves as rational creatures, and the next, in point of importance, as citizens, is that, which includes so many, of a mother ».

95. Ibid., p. 450 : « asserting the rights which women in common with men ought to contend for, I have not attempted to extenuate their faults; but to prove them to be the natural consequence of their education and station in society. If so, it is reasonable to suppose that they will change their character, and correct their vices and follies, when they are allowed to be free in a physical, moral, and civil sense ».

96. Regina M. JANES, «On the Reception of Mary Wollstonecraft's: A Vindication of the Rights of Woman », Journal of the History of Ideas, 39, 2, 1978, p. 293-302. 
97. Analytical Review, 12, 1792, p. 241-49; 13, 1792, p. 481-89.

98. General Magazine, 6, 1792, p. 187-91.

99. Literary Magazine, 1, 1792, p. 133-39.

100. Mary WOLLSTONECRAfT, A Vindication of the Rights of Woman: with Strictures on Political and Moral Subjects, Boston, Peter Edes, 1792.

101. ID., Défense des droits des femmes, suivie de quelques considérations sur des sujets politiques et moraux, ouvrage traduit de l'anglais, Paris, Buisson, 1792.

102. ID., An Historical and Moral View of the Origin and Progress of the French Revolution and the effect it has produced in Europe, Londres, Johnson, 1795.

103. Horace Walpole's Correspondence with Hannah More, et alii, éd. par W. S. Lewis, Robert A. Smith, Charles H. Bennett, Londres, Oxford University Press, 1961, vol. 31, p. 370, 373, 397.

104. Ibid., p. 7 : « nature having made men unequal, by giving stronger bodily and mental powers to one than to another, the end of government ought to be, to destroy this inequality by protecting the weak ».

105. Sur l'interprétation philosophique qui sous-tend cet ouvrage, voir Virginia SAPIRO, A Vindication of Political Virtue: The Political Theory of Mary Wollstonecraft, Chicago, University of Chicago Press, 1992 et Isabelle BoUR, « Mary Wollstonecraft as Historian in An Historical and Moral View of the Origin and Progress of the French Revolution; and the Effect it has Produced in Europe (1794)", Études Épistémè, $\mathrm{n}^{\circ}$ 17, 2010, https://journals.openedition.org/episteme/668.

106. Ibid., p. 221 : «the most arbitrary governments in Europe, Russia excepted, begin to treat their subjects as human beings, feeling like men, and with some powers of thinking ».

107. Ibid., p. 234 : "the natural and imprescriptible right of man; without the enjoyment of which, it is impossible for him to become either a reasonable or dignified being ".

108. Ibid., p. 201 : «the almost incredible exertion of an indignant people; who felt, for the first time, that they were sovereign, and that their power was commensurate to their will».

109. Ibid., p. 200.

110. Ibid., p. 311 : « have not those factitious, supercilious manners, common to the english; and acting more freely, they have more decision of character, and even more generosity ».

111. Ibid., p. 425 : «From the enjoyment of more freedom than the women of other parts of the world, those of France have acquired more independence of spirit than any others ; it has, therefore, been the scheme of designing men very often since the revolution, to lurk behind them as a kind of safeguard, working them up to some desperate act, and then terming it a folly, because merely the rage of women, who were supposed to be actuated only by the emotions of the moment. Early then on the fifth of October a multitude of women by some impulse were collected together; and hastening to the hotel-de-ville obliged every female they met to accompany them, even entering many houses to force others to follow in their train ».

112. Ibid., p. 426 : " mostly of market women, and the lowest refuse of the streets, women who had thrown off the virtues of one sex without having power to assume more than the vices of the other».

113. Ibid. : " not to be confounded with the honest multitude who took the Bastille ».

114. Ibid., p. 453 : that a body of women should put themselves in motion to demand relief of the king, or to remonstrate with the assembly respecting their tardy manner of forming the constitution, is scarcely probable ».

115. Ibid., p. 247 : « These sudden transitions from one extreme to another, without leaving any settled conviction behind, to confirm or eradicate the corroding distrust, could not be seen in such a strong light anywhere as at Paris, because there a variety of causes have so effeminated reason, that the French may be considered as a nation of women ».

116. Ibid., p. 469 : «Freedom is a solid good, that requires to be treated with reverence and respect. But, whilst an effeminate race of heroes are contending for her smiles, with all the 
blandishments of gallantry, it is to their more vigorous and natural posterity, that she will consign herself with all the mild effulgence of artless charms ».

117. Mary WOLLSTONECRAFT, "Compte-rendu de Helen Maria Williams, Letters written in France, in the summer 1790, to a Fiend in England", dans Janet Todd et Marilyn Butler (ed.), The Works of Mary Wollstonecraft, op. cit, vol. 7, p. 322.

118. ID., "Compte-rendu de Catherine Macaulay, Letters on Education: with Observations on Religious and Metaphysical Subjects", dans Janet Todd et Marilyn Butler (ed.), The Works of Mary Wollstonecraft, op. cit, vol.7, p. 309: «this masculine and fervid writer has turned the very superior powers of her mind to the consideration of a subject, which, perhaps embraces a wider circle of unsettled opinions ".

119. ID., A Vindication of the Rights of Woman, op. cit., p. $4:$ « the imitation of manly virtues, or, more properly speaking, the attainment of those talents and virtues, the exercise of which ennobles the human character, and which raise females in the scale of animal being, when they are comprehensively termed mankind ".

120. Linda Colley, Britons. Forging the Nation 1707-1837, Londres, Pimlico, 2003 [1992], p. 252.

121. Mary wollstonecraft, An Historical and Moral View of the Origin and Progress of the French Revolution, op. cit., p. 35 : «the dupe of the knaves she fostered».

122. Helen Maria WiLliams, Lettres sur les événemens qui se sont passés en France, op. cit., p. 2.

123. Deborah KENNEDY, «Spectacle of the Guillotine: Helen Maria Williams and the Reign of Terror ", Philological Quarterly, Winter 1994, p. 95-113 : 97.

124. Lynn HUNT, Politics, Culture and Class in the French Revolution, Berkeley, University of California Press, 1986, p. 94-98.

125. Helen Maria WiLliams, Letters Containing a Sketch of the Scenes Which Passed in Various Departments of France During the Tyranny of Robespierre op. cit., p. 122 : « when she received the fatal stroke, the streams of maternal nourishment issued rapidly from her bosom, and mingled with her blood, bathed her executioner $"$.

126. Ibid., p. 19.

127. Ibid., p. 48 : « he took up this young girl, crawling for weakness at her mother's feet; while her sisters, being a few years older, were doomed to perish. But to perish together now seemed their only consolation; they refused to part; and some degree of violence was used to force away the child; who soon after the murder of her family died of a broken heart ».

128. Helen Maria WiLliams, Lettres sur les événemens qui se sont passés en France op. cit., p. 171.

129. Ibid., p. 172.

130. Ibid.

131. John BARRELL \& Jon MEE, Trials for treason and sedition, 1792-1794, Londres, Pickering \& Chatto, 2006. Michael LobBAn, "Treason, Sedition and the Radical Movement in the Age of the French Revolution ", Liverpool Law Review, 22, 2000, p. 205-234.

132. Helen Maria WiLliams, Lettres sur les événemens qui se sont passés en France op. cit., p. 64.

133. Ibid., p. 104.

134. Ibid., p. 105.

135. Ibid., p. 96.

136. Ibid., p. 98.

137. Ibid., p. 100.

138. Ibid., p. 156.

139. Ibid., p. 157.

140. Sur le terme "monstruosité sanguinaire " que Williams utilise à plusieurs reprises pour décrire les actions de Robespierre et de sa faction, Elizabeth Bohls a noté, outre à un renvoi à la rhétorique post-thermidorienne, une référence au roman gothique: Elizabeth BOHLS, Women 
Travel Writers and the Language of Aesthetics, 1716-1818, Cambridge, Cambridge University Press, 1995, p. 130.

141. Helen Maria WiLliams, Lettres sur les événemens qui se sont passés en France, op. cit., p. 157.

142. ID., Sketches of Manners and Opinions in the French Republic towards the Close of the Eighteenth Century in a Series of Letters, 2 vol., Londres, Robinson, 1801.

143. ID., Aperçu de l'état des mœurs et des opinions dans la république française, vers la fin du XVIII siècle, 2 vol., Paris, chez les frères Levarult, 1801.

144. Pour une analyse précise de cet ouvrage, voir Caroline FRANKLIN, «'The colour of a riband': Patriotism, history and the role of women in Helen Maria Williams's Sketches of Manners and Opinions in the French Republic (1801) ", Women's writing, 13, 3, 2006, p. 495-508.

145. Helen Maria wiLliams, Aperçu de l'état des mœurs et des opinions dans la république française, op. cit., p. XII.

146. Ibid., p. XIII.

147. Ibid., p. 43.

148. Ibid.

149. Ibid., p. 46.

150. Sur le rapport entre Révolution française et révolution de Naples et les sources de Williams, voir Anna Maria RAO, Esuli: l'emigrazione politica italiana in Francia (1792-1802), Naples, Guida editori, 1992, p. 441-464.

151. Helen Maria williams, Aperçu de l'état des mœurs et des opinions dans la république française, op. cit., vol. 2, p. 46.

152. Ibid., p. 47.

153. Ibid., p. 48.

154. Ibid., p. 50.

155. Charles Guillaume tHÉREMIN, De la condition des femmes dans les républiques, Paris, Chez Laran, an VII-1799.

156. Helen Maria WiLliams, Aperçu de l'état des mœurs et des opinions dans la république française, op. cit, vol. 2, p. 53.

157. Sur l'activité de Williams sous le Consulat voir Paolo CONTE, «Comment (et pourquoi) parler de Révolution sous le Consulat: la Correspondance politique et confidentielle inédite de Louis XVI, par Helen Maria Williams ", La Révolution française, $\mathrm{n}^{\circ} 15,2018$, https://doi.org/ 10.4000/lrf.2400.

158. Helen Maria WiLliams, Aperçu de l'état des mœurs et des opinions dans la république française, op. cit, vol. 2, p. 59.

159. Sur le rôle de Williams comme médiatrice culturelle, voir Bernard GAINOT, «Helen-Maria Williams, médiatrice culturelle dans La Décade philosophique », La Révolution française, $\mathrm{n}^{\circ} 12,2017$, https://doi.org/10.4000/lrf.1754.

160. Hannah MORE, Village Politics: Addressed to all the Mechanics, Journeymen, and Day Labourers, in Great Britain. By Will Chip, a Country Carpenter, Manchester, J. Harrop, 1793.

\section{RÉSUMÉS}

Le bouleversement de la Révolution française a secoué l'Europe entière. La société britannique est sans doute celle qui a le plus ressenti les conséquences de ces événements. Parmi les 
nombreux Anglais qui se sont interrogés sur l'impact et les conséquences de la Révolution, sur qui l'historiographie depuis longtemps se concentre, Mary Wollstonecraft et Helen Maria Williams constituent un cas d'étude particulièrement intéressant. Elles n'ont pas seulement joué un rôle important dans l'interprétation de la Révolution, mais aussi sur le front de la participation en décidant de s'installer à Paris pour voir de leurs propres yeux les événements de France. Étudier comment Mary Wollstonecraft et Helen Williams ont décrit et vécu la Révolution française permet de réfléchir à la construction du récit de la Révolution au prisme de leur regard de femmes et d'étrangères et de revendiquer l'agentivité de celles qui, comme Wollstonecraft et Williams, ont voyagé, écrit et pris la parole pour défendre leurs droits.

The upheaval of the French Revolution shook the whole Europe. British society is arguably the one that felt the consequences of these events the most. Among the many English writers who questioned the impact and consequences of the Revolution, on whom historiography has long focused, Mary Wollstonecraft and Helen Maria Williams constitute a particularly interesting case study. They not only played an important role in the interpretation of the Revolution, but also on the participation front by deciding to settle in Paris to see for themselves the events in France. Studying how Mary Wollstonecraft and Helen Williams described and experienced the French Revolution allows us to reflect on the construction of the history of the Revolution through the gaze of women and strangers and to claim the agency of those, like Wollstonecraft and Williams, who have travelled, written, and spoken to defend their rights.

\section{INDEX}

Keywords : Mary Wollstonecraft, Helen Maria Williams, Women writers, Women, French Revolution, English writers, Dissenter, Women's rights

Mots-clés : Mary Wollstonecraft, Helen Maria Williams, Femmes de lettres, Femmes, Révolution, Anglaises, Dissenter, Étrangères, Droits des femmes

\section{AUTEUR}

\section{VALENTINA ALTOPIEDI}

IHMC-IHRF, Université de Turin 